\title{
Is symmetry inference an essential component of language?
}

\author{
Thomas F. Chartier ${ }^{1}$ - Arnaud Rey ${ }^{1}$ \\ Published online: 15 January 2020 \\ (C) The Psychonomic Society, Inc. 2020
}

\section{Summary}

Symmetry inference - that is, spontaneously deriving the stimulus association B-A from A-B - was recently reported in preverbal infants (Kabdebon \& Dehaene-Lambertz, 2019, Proceedings of the National Academy of Sciences of the United States of America, 116[12], 5805-5810) and regarded as a "building block for human cognition." Here, we argue that empirical evidence supporting this claim is insufficient, and that absence of symmetry inference in nonhuman animals should be reassessed.

Keywords Associative learning $\cdot$ Symmetry inference $\cdot$ Preverbal humans $\cdot$ Language evolution

Bidirectional associations are a central feature of human language, as they permit a flexible and interchangeable use between spoken, written, or sign words, and representations of the objects referred to. For example, one can both think about a dog upon hearing "dog" and say "dog" upon thinking about one. While such symmetrical associations are easily conceived as arising from a bidirectional training, as happens when parents repeatedly name and show a given object to a child, human adults or children trained on successive stimulus pairings A-B have been reported to spontaneously derive the reversed, untrained relations B-Athat is, to infer bidirectional associations from unidirectional ones (e.g., Sidman et al., 1982). This capacity, called symmetry inference, has proved highly difficult to demonstrate experimentally in nonhuman animals (e.g., Medam, Marzouki, Montant, \& Fagot, 2016) and could thus be unique to people. Such findings have raised strong interest as to the potential necessity of symmetry inference for language to develop, making it the marker of a possible discontinuity between human and nonhuman behavior.

However, because symmetry inference means disregarding the order of elements that are associated, one can argue that such propensity can be detrimental, as it may break naturally occurring causal regularities and induce maladaptive behaviors. In this view, it is expected that nonhuman animals do not show symmetry inference, and surprising that humans do; such cognitive bias in humans might simply emerge together with language development, as a by-product of our constant use of bidirectional associations.

A strong argument in favor of symmetry inference being required for language would be to find it in preverbal humans.

Arnaud Rey

arnaud.rey@univ-amu.fr

1 Laboratoire de Psychologie Cognitive, CNRS \& Aix-Marseille Université, UMR 7290, Bâtiment 9 Case D, 3, place Victor Hugo, 13331 Marseille Cedex 3, France
A recent PNAS article (Kabdebon \& Dehaene-Lambertz, 2019) has reported such results. Using EEG recordings, the authors tested the ability of 5-month-old infants to abstract representations from patterns of trisyllabic spoken nonsense words (e.g., "ba-ke-tu") and associate these representations with visual or auditory labels (e.g., a picture of a lion). In their critical Experiment 3, upon testing 34 infants with reversed pairs (i.e., presenting them with label-pattern pairs after a pattern-label training), the authors observed different brain activities depending on whether reversed pairs were consistent or inconsistent with initial pairings. They interpret this as evidence that infants expected the second stimuli (the patterns) consistently with training, meaning that they had inferred symmetrical associations from unidirectional ones. The authors view this ability as "a foundational operation for any symbolic system, p. 5805" and, arguing that other animals do not have it, they suggest that it constitutes "a building block for human cognition and notably language development, p. 5808" distinguishing humans from nonhumans. We would like to argue that both arguments supporting this claimnamely, that preverbal humans can infer symmetry, and that nonhumans cannot - are insufficiently grounded.

First, the data interpretation in their study is certainly debatable. Contrary to their claim, a careful inspection of the experimental design reveals that the trained associations were already bidirectional, and consequently, infants did not need to infer symmetry. The flaw, present in all three experiments, was twofold. During the training period, infants were not exposed to well separated pattern-label pairs, but with a regularly alternating sequence of word patterns and labels. Indeed, and surprisingly, the intertrial stimulus interval (ITI $=733 \mathrm{~ms}$ ) was comparable to the within-trial stimulus interval $(980 \mathrm{~ms})$. Consequently, infants experienced a similar temporal contiguity for the backward associations label-pattern between two consecutive pairs as for the forward associations pattern-label of each pair, and had the opportunity to learn both types of relations. The 
artificial separation of trials by a distracting visual stimulus (the blinking eyes) could not prevent such learning from taking place, as infants are known to be particularly sensitive to statistical structure in temporal sequences. This need not have been a problem, had a given label not systematically been followed by the same word pattern. Yet precisely this happened, as pairs were repeated in 12 consecutive trials (see their Supplementary Material, p. 5). To illustrate, if one calls P and $\mathrm{Q}$ the word patterns and $\mathrm{X}$ and $\mathrm{Y}$ the labels, infants were effectively first exposed to PXPXPX . . . PX (12 times) then QYQYQY ... QY (12 times) - that is, almost as many reversed instances of the consistent pairs (XP and YQ, 11 times) as forward instances (PX and QY, 12 times). The subsequent randomized presentation of pairs was too brief to correct for the statistical structure already introduced: PX and QY each randomly appearing six times yielded exposition to reversed consistent (XP and YQ) and reversed inconsistent (XQ and YP) pairs, both on average three times, and at most six. Consequently, not only were infants explicitly exposed to reversed pairs before testing, but this happened with more consistent ones (around 14 times) than inconsistent ones (around three times). Not surprisingly, when the test was performed (Experiment 3), a differential brain response was detected between consistent and inconsistent reversed pairings. The data thus reveal only a simultaneous learning of several conditional associations and do not allow us to conclude that 5-month-old infants can infer symmetry. Adequate experiments would require a familiarization phase with a longer ITI and a fully randomized presentation of pairs.

No other study, to our knowledge, has searched for symmetry inference in preverbal humans, hence the important question of whether such capacity develops before language use remains unanswered. To address it, investigations in preverbal infants are crucial, because symmetry inference in verbal subjects is easily accounted for in terms of verbal recoding. Since human subjects readily name experimental stimuli, the phonological loop is likely used, which naturally transforms unidirectional associations into bidirectional ones.

Second, despite plentiful negative results, some words of caution are needed before asserting that symmetry inference is only observed in humans. A familiarity with symmetry, as verbal humans always have, may simply be needed. Indeed, one study including a symmetry training on a subset of stimulus pairs revealed symmetry inference in a sea lion (Schusterman \& Kastak, 1993). Besides, to our knowledge, symmetry inference in humans was never demonstrated with a strictly unidirectional training. All studies (e.g., Sidman et al., 1982) have used identity training (i.e., have included identical pairs A-A and B-B alongside arbitrary pairs A-B, to avoid the surprise effects of seeing items $A$ and $B$ in new ordinal positions during the test with B-A). Adding such training in pigeons also yielded evidence for symmetry inference (Frank \& Wasserman, 2005). Hence, at least two experimental conditions promote symmetry inference in nonhumans - one of which may even be required in humans. Furthermore, all studies so far have looked for a spontaneous motor output exemplifying the reversed stimulus pairings. But it might be that animal subjects do infer symmetry, though for some reason they do not show explicit signs of it. Consequently, implicit signatures of symmetry inference, for example, a faster learning of reversed pairs compared with arbitrary pairs, should also be looked for. The purported absence of symmetry inference in nonhuman animals should thus be reassessed, with new comparative experiments examining the influence of both symmetry training and identity training on symmetry inference, and including implicit tests.

Clarifying both major questions - whether symmetry inference is found only in humans and whether it develops before language - will lead to one of four outcomes: (1) If it is indeed absent in nonhumans and present in preverbal humans, as speculated in the PNAS study, then it could constitute a key prerequisite for language. Yet proving that it is required would still be difficult, as one would typically need to somehow suppress this ability and show that language development is impaired. (2) If both nonhumans and preverbal humans can infer symmetry, then it could still represent a crucial, though not sufficient, building block of language. (3) If symmetry inference is unique to humans and develops only after language, it would simply be a bias stemming from language, allowing for logically wrong inferences. (4) If instead it is shared with nonhumans, though this is less likely, it would be a capacity independent of language.

Finally, it is probably important to distinguish between inferential and processing abilities. Nonhuman animals may be unable to infer reverse associations they have not experienced, while nevertheless using bidirectional associations which they would learn through simultaneous stimulus exposure (i.e., through bidirectional, not unidirectional training).

\section{References}

Frank, A. J., \& Wasserman, E. A. (2005). Associative symmetry in the pigeon after successive matching-to-sample training. Journal of the Experimental Analysis of Behavior, 84(2), 147-165. https://doi.org/ 10.1901/jeab.2005.115-04

Kabdebon, C., \& Dehaene-Lambertz, G. (2019). Symbolic labeling in 5month-old human infants. Proceedings of the National Academy of Sciences of the United States of America, 116(12), 5805-5810. doi: https://doi.org/10.1073/pnas.1809144116

Medam, T., Marzouki, Y., Montant, M., \& Fagot, J. (2016). Categorization does not promote symmetry in Guinea baboons (Papio papio). Animal Cognition, 19(5), 987-998. doi:https://doi. org/10.1007/s10071-016-1003-4

Schusterman, R. J., \& Kastak, D. (1993). A California sea lion (Zalophus californianus) is capable of forming equivalence relations. Psychological Record, 43(4), 823-839. https://doi.org/10.1007/BF03395915

Sidman, M., Rauzin, R., Lazar, R., Cunningham, S., Tailby, W., \& Carrigan, P. (1982). A search for symmetry in the conditional discriminations of rhesus monkeys, baboons, and children. Journal of the Experimental Analysis of Behavior, 37(1), 23-44. https://doi.org/10.1901/jeab.1982.37-23

Publisher's note Springer Nature remains neutral with regard to jurisdictional claims in published maps and institutional affiliations. 